\title{
Magnetic interaction of jets and molecular clouds in NGC 4258
}

\author{
M. Krause ${ }^{1}$, C. Fendt ${ }^{2}$, and N. Neininger ${ }^{1,3,4}$
}

\author{
1 Max-Planck-Institut für Radioastronomie, Auf dem Hügel 69, 53121 Bonn, Germany \\ e-mail: mkrause@mpifr-bonn.mpg.de \\ 2 Max-Planck-Institut für Astronomie, Königstuhl 17, 69117 Heidelberg, Germany \\ 3 Radioastronomisches Institut der Universität Bonn, Auf dem Hügel 71, 53121 Bonn, Germany \\ ${ }^{4}$ Institut de Radioastronomie Millimétrique, 300 rue de la Piscine, 38406 St. Martin d'Hères, France
}

Received 26 September 2006 / Accepted 9 March 2007

\section{ABSTRACT}

\begin{abstract}
Context. NGC 4258 is a well known spiral galaxy with a peculiar large scale jet flow detected in the radio and in $\mathrm{H} \alpha$. Due to the special geometry of the galaxy, the jets emerge from the nuclear region through the galactic disk - at least in the inner region. Aims. Also the distribution of molecular gas looks different from that in other spiral galaxies: ${ }^{12} \mathrm{CO}(1-0)$ emission has only been detected in the center and along the jets and only up to distances of about $50^{\prime \prime}(1.8 \mathrm{kpc})$ from the nucleus. This concentration of $\mathrm{CO}$ along the jets is similar to what is expected as fuel for jet-induced star formation in more distant objects. The reason for the CO concentration along the inner jets in NGC 4258 was not understood and is the motivation for the observations presented here. Methods. Using the IRAM interferometer at Plateau de Bure, we mapped the ${ }^{12} \mathrm{CO}(1-0)$ emission of the central part of NGC 4258 along the nuclear jet direction in the inner $3 \mathrm{kpc}$. In order to get a properly positioned overlay with $\mathrm{H} \alpha$ we observed NGC 4258 in $\mathrm{H} \alpha$ at the Hoher List Observatory of the University of Bonn.

Results. We detected two parallel CO ridges along a position angle of $-25^{\circ}$ with a total length of about $80^{\prime \prime}$ ( $\left.2.8 \mathrm{kpc}\right)$, separated by a CO depleted funnel with a width of about $5^{\prime \prime}(175 \mathrm{pc})$. The $\mathrm{H} \alpha$ emission is more extended and broader than the CO emission with its maximum just in between the two CO ridges. It seems to be mixed in location and in velocity with the $\mathrm{CO}$ emission. In $\mathrm{CO}$ we see a peculiar velocity distribution in the iso-velocity map and $\mathrm{p}-\mathrm{v}$ diagrams. We discuss different scenarios for an interpretation and present a model which can explain the observational results consistently.

Conclusions. We propose here that the concentration of $\mathrm{CO}$ along the ridges is due to interaction of the rotating gas clouds with the jet's magnetic field by ambipolar diffusion (ion-neutral drift). This magnetic interaction is thought to increase the time the molecular clouds reside near the jet thus leading to the quasi-static $\mathrm{CO}$ ridge.
\end{abstract}

Key words. galaxies: active - galaxies: individual: NGC 6445 - galaxies: jets - magnetic fields - galaxies: ISM - radio lines: galaxies

\section{Introduction}

The nearby $(D=2 \mathrm{Mpc})$ spiral galaxy NGC 4258 is known for its anomalous $\mathrm{H} \alpha$ and non-thermal radio arms located in between the normal spiral arms (Courtès \& Cruvellier 1961; van der Kruit et al. 1972). The anomalous arms are usually explained in terms of collimated nuclear outflows or jets emerging from the nuclear region. In the central region, there is a bending at $70^{\prime \prime}(2.5 \mathrm{kpc})$ radial distance in a trailing sense with respect to the galactic rotation. The interpretation in terms of nuclear jets is consistent with the detection of strong nuclear maser emission from an edge-on, east-west orientated nuclear accretion disk (Watson \& Wallin 1994; Greenhill et al. 1995) with a super massive nuclear engine (Miyoshi et al. 1995).

Cecil et al. (1995a,b) presented a high resolution radio continuum map which shows the central radio emission to be nearly perpendicular to the nuclear disk (Table 1) as expected for jets. The radio jet can even be followed to mas scale with VLBA observations (Herrnstein et al. 1997, 1998a, 2005).

The geometical parameters of the galactic and the nuclear disk and the jet features of NGC 4258 are summarized in Table 1. As the kpc-jet axis lies nearly along the major axis of the disk, the jets have to pass through the galactic disk, at least in the central part, and an interaction with the interstellar medium (ISM) of the disk of NGC 4258 is expected.
Cecil et al. (1992) identified the jets in the SE in the inner $2 \mathrm{kpc}$ as a helical, braided structure of 3 intertwined plasma streams. The emission line velocity field suggests that the gas moves along the helices. Already these authors assumed an interaction of the jets with the ISM of the galaxy.

Molecular gas $\left({ }^{12} \mathrm{CO}(1-0)\right)$ in NGC 4258 has first been observed with the Kitt Peak 12-m telescope (Adler \& Liszt 1989), with the Nobeyama 45-m telescope (Sofue et al. 1989), and with the Owens Valley three element interferometer (OVRO)(Martin et al. 1989). While the Kitt Peak observations detected CO in the central $60^{\prime \prime}$ but lack angular resolution to show details, the spatial and intensity distribution of the Nobeyama observations turned out to be not reliable enough. With the OVRO Martin et al. (1989) resolved the central emission in two components lying on each side of the nucleus and outlining a narrow channel through the interstellar disk corresponding to the $\mathrm{H} \alpha$ jets. Entrained and shocked gas of the ISM were proposed to be responsible for the optical line emissions. X-ray observations of the jets are interpreted by Cecil et al. $(1995 \mathrm{a}, \mathrm{b})$ as due to hot, shocked gas from nearby molecular clouds that have been entrained into the jet.

The OVRO data were further analyzed by Plante et al. (1991) who suggested that the $\mathrm{CO}$ distribution confines the inner part of the $\mathrm{H} \alpha$ jets and that the line-emitting jet may even be deflected by a dense molecular cloud. Cecil et al. (2000) claimed to have 
Table 1. Geometrical parameters of NGC 4258.

\begin{tabular}{lll}
\hline \hline Galaxy & & \\
Morphological type & $\mathrm{SAB}(\mathrm{s}) \mathrm{bc}$ & $(1)$ \\
Distance & $7.2 \pm 0.3 \mathrm{Mpc}$ & $(2)$ \\
& $\left(1^{\prime \prime}\right.$ corresponds to $\left.35 \mathrm{pc}\right)$ & \\
Inclination & $72^{\circ}$ & $(3)$ \\
$\begin{array}{l}\text { Position angle (PA) } \\
\text { of major axis }\end{array}$ & $-30^{\circ}$ & $(3)$ \\
PA of HI bar & $10^{\circ} \pm 3^{\circ}$ & $(3)$ \\
Nuclear disk & & \\
Inclination & $83^{\circ} \pm 4^{\circ}$ & $(4)$ \\
Position angle & $86^{\circ} \pm 2^{\circ}$ & $(4)$ \\
Jet features & & \\
PA in radio continuum & $-3^{\circ} \pm 1^{\circ}$ for $d<24^{\prime \prime}$ & $(5,6)$ \\
& $-43^{\circ} \pm 1^{\circ}$ for $d>24^{\prime \prime}$ & $(6)$ \\
PA in H $\alpha$, CO & $-25^{\circ}$ fo $d<24^{\prime \prime}$ & $(7)$ \\
$\beta$ in radio continuum & $60^{\circ}$ for \\
& $75^{\circ}$ for $d>24^{\prime \prime}$ & $(7,8)$ \\
$\beta$ in H $\alpha$, CO & $83^{\circ}$ \\
\hline
\end{tabular}

(1) de Vaucouleurs et al. (1976); (2) Herrnstein et al. (1999); (3) van Albada (1980); (4) Miyoshi et al. (1995); (5) Cecil et al. (2000); (6) Krause \& Löhr (2004); (7) this paper; (8) $\beta$ is the angle between the nuclear jet axis and the rotational axis of the galaxy as discussed in Sect. 4.2 adopting the warped disk model by Herrnstein et al. (2005) with a central disk inclination of $74^{\circ}$.

detected two radio hot spots 24 " north and $49^{\prime \prime}$ south of the nucleus and inferred that the jets may have precessed on a large cone through the disk plane. The interpretation of these two radio sources as being hot spots is, however, still under debate as the northern one has been classified as SNR candidate by Hyman et al. (2001); Krause \& Löhr (2004) detected linear polarization in the northern one but not in the southern.

The first reliable single-dish observations with enough angular resolution to resolve the ${ }^{12} \mathrm{CO}(1-0)$ emission of NGC 4258 had been carried out with the 30-m IRAM telescope at Pico Veleta (Krause et al. 1990). Molecular gas in NGC 4258 has only been detected in the center and along the $\mathrm{H} \alpha$ jets, at distances up to $2 \mathrm{kpc}$ (Krause et al. 1990). It has not been found in other parts of the (central) galactic disk up to the detection limit of about $30 \mathrm{mK}$. Observations by Cox \& Downes (1996) confirm this.

In this paper we present high resolution Plateau de Bure (PdB) interferometer measurements of the molecular gas that were combined with single-dish data of the 30-m IRAM telescope. Hence, we present the ${ }^{12} \mathrm{CO}(1-0)$ observations with the highest angular resolution $\left(4\right.$ ". $\left.^{\circ} \times 33^{\prime \prime} 3\right)$ obtained so far from NGC 4258 without lacking for the extended emission due to missing spacings from interferometer data alone. In the meantime, Helfer et al. (2003) published maps of the CO emission of NGC 4258 as part of the BIMA Survey of 44 nearby galax-

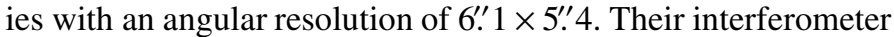
observations were combined with the 12-m NRAO telescope on Kitt Peak. Their maps agree well with our results. These data were recently analysed together with ${ }^{12} \mathrm{CO}(2-1)$ interferometer observations taken with the Submillimeter Array (SMA) on Mauna Kea by Sawada-Satoh et al. (2007). They also interpret their data as indicating interaction of the molecular gas by expanding motions from the nuclear region.

In Sect. 2 we will describe our observations in ${ }^{12} \mathrm{CO}(1-0)$ and in $\mathrm{H} \alpha$ and present the observational results in Sect. 3. The discussion follows in Sect. 4 and Sect. 5 contains the summary and conclusions.

\section{Observations}

We observed the central region of NGC 4258 along the inner $\mathrm{H} \alpha$ jets up to a radial distance of about $2 \mathrm{kpc}$ in ${ }^{12} \mathrm{CO}(1-0)$ using the Plateau de Bure $(\mathrm{PdB})$ interferometer in its compact configuration, i.e. with baselines ranging from $24 \mathrm{~m}$ to $176 \mathrm{~m}$. In order to account for the elongated shape of the central region we set up a mosaic consisting of 5 fields that were aligned with the $\mathrm{CO}$ ridge known from previous single-dish observations (Krause et al. 1990). The central and reference position of our observations is $\alpha_{2000}=12^{\mathrm{h}} 18^{\mathrm{m}} 57^{\mathrm{s}} 55, \delta_{2000}=47^{\circ} 18^{\prime} 14.2^{\prime \prime}$. The spacing between the individual pointings was half the primary beam width (FWHM). The positions relative to the center of the 5 fields of the mosaic are $\left(0^{\prime \prime}, 0^{\prime \prime}\right),\left(10^{\prime \prime},-20^{\prime \prime}\right),\left(18^{\prime \prime},-40^{\prime \prime}\right)$, $\left(-10^{\prime \prime}, 20^{\prime \prime}\right),\left(-18^{\prime \prime}, 40^{\prime \prime}\right)$. These positions agree within $2^{\prime \prime}$ with the major axis of NGC 4258. Thus, we covered with our mosaic an area of $40^{\prime \prime} \times 100^{\prime \prime}$, with a uniform sensitivity along the major axis of the resulting oval. The five fields were pointed at sequentially, spending 4 min for each pointing; after 20 min the phase calibrator was observed and the sequence started again.

The correlator backends were set such as to cover the whole IF bandpass with a resolution of $2.5 \mathrm{MHz}\left(6.3 \mathrm{~km} \mathrm{~s}^{-1}\right)$ plus two units that were set to cover the central velocity range with a resolution of $0.625 \mathrm{MHz}$.

The flux and bandpass calibration was determined by observing 3C273 and $\mathrm{W} 3 \mathrm{OH}$. We used $1044+719$ as phase calibrator. The 5 fields were combined in a mosaic and subsequently cleaned using the MAPPING procedure of the GILDAS software package. The maps were cleaned with varied clean boxes for the different channels according to their intensity distribution.

We corrected the data for "missing short spacings" by combining them with single dish data obtained with the 30-m telescope at Pico Veleta by Cox \& Downes (1996). For the combination we used the "ssc method" as described by Weiss et al. (2001) that works on the finally reduced (cleaned and corrected for primary beam attenuation) interferometer cube. Both data sets were transformed into the uv plane using an FFT algorithm. The inner visibilities of the interferometer data were replaced with the single-dish values. The angular resolution of the final maps is $4^{\prime \prime} .6 \times 33^{\prime \prime} 3$ with a position angle of $31^{\circ}$.

We further observed NGC 4258 in $\mathrm{H} \alpha$ at the Hoher List Observatory of the University of Bonn. The WWFPP focal reducer/CCD camera at the 1-m telescope gives a field of view of $20^{\prime}$ diameter at $0.8^{\prime \prime}$ pixel size. The seeing was about $2^{\prime \prime}$ and the integration time in the $4 \mathrm{~nm}$ wide $\mathrm{H} \alpha$ filter $32 \mathrm{~min}$. A broadband red image was taken at the same time to subtract the continuum emission. The large number of cataloged stars in the field ensures a proper relative positioning of the $\mathrm{H} \alpha$ and the $\mathrm{CO}$ emission with an error of the order of $1^{\prime \prime}$.

\section{Observational results}

\subsection{The CO spectra and channel maps}

We received spectra ranging from -250 to $+250 \mathrm{~km} \mathrm{~s}^{-1}$ with respect to the systemic velocity $v_{\mathrm{lsr}}$ of $457 \mathrm{~km} \mathrm{~s}^{-1}$ (van Albada 1980). Peak intensities reach about $300 \mathrm{mJy} / \mathrm{beam}$ in the strongest spectra. Many of the spectra have more than one velocity component (see Sect. 3.3).

The central position of our observations (as given in Sect. 2) agrees within less than 1 " with the position of the central maser 


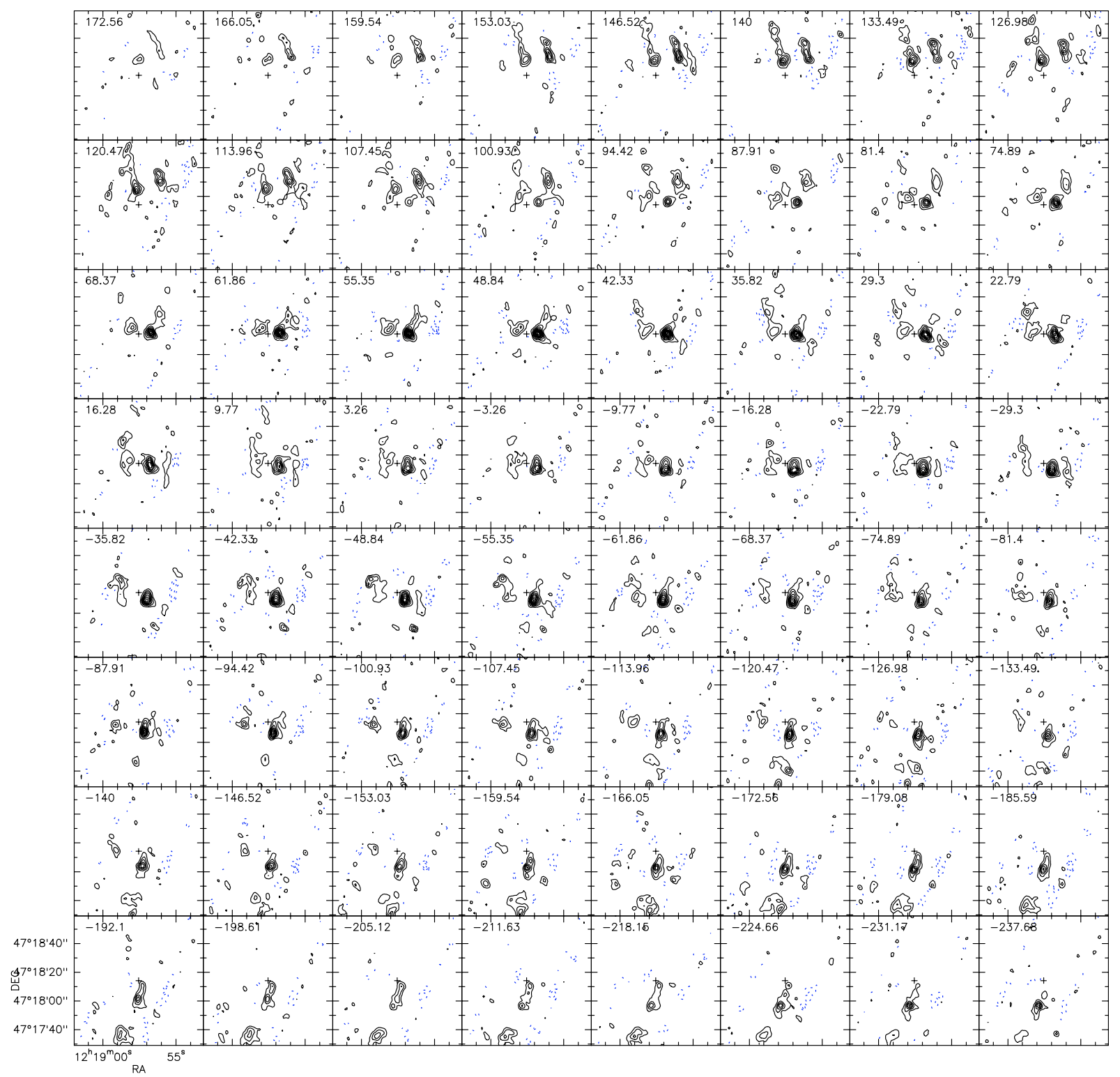

Fig. 1. Channel maps of ${ }^{12} \mathrm{CO}(1-0)$ of NGC 4258 . The contour levels are $50 \mathrm{mJy} / \mathrm{beam}$. The velocity is given in the upper left corner in each panel.

emission as determined by Greenhill et al. (1995). We could not detect $\mathrm{CO}$ right at the nucleus. In general, there is a gap in the $\mathrm{CO}$ distribution along the central axis of the inner $\mathrm{H} \alpha$ jet. This is also obvious in the channel maps shown in Fig. 1. All panels in the velocity range between -160 to $+160 \mathrm{~km} \mathrm{~s}^{-1}$ show two main blobs oriented in east west direction and separated by $15^{\prime \prime}$ to $20^{\prime \prime}$. The positions of the blobs shift together from north to south when changing the velocities from positive to negative values.

\subsection{Integrated CO intensity and comparison with $\mathrm{H} \alpha$}

We determined the zeroth moments by integrating the line intensity in the individual spectra. All points with values below 3 times the noise level $\left(\sigma=0.7 \mathrm{Jy} /\right.$ beam $\left.\mathrm{km} \mathrm{s}^{-1}\right)$ of the inner part of the map were discarded in the calculation. The spatial distribution of the integrated $\mathrm{CO}$ emission in the observed area is shown in Fig. 2.
The molecular gas follows two parallel ridges along a position angle of about $-25^{\circ}$. The western ridge is stronger in intensity and with a total projected length of about $80^{\prime \prime}$ more extended than the eastern one. The width of each ridge $(H P B W)$ is about $7^{\prime \prime}(250 \mathrm{pc})$. The ridges are separated by about $5^{\prime \prime}(175 \mathrm{pc})$, a region with strong $\mathrm{CO}$ depletion. The orientation of the ridges agrees well with previous $\mathrm{CO}$ observations. (They had however either not enough spatial resolution to separate both ridges (Krause et al. 1990; Cox \& Downes 1996) or not enough sensitivity to trace both ridges (Plante et al. 1991, 1994).) This orientation matches the mean jet direction in the radio continuum up to a distance of about $1.5^{\prime}$ from the nucleus (see Table 1).

Both northern ridges seem to kink by about $30^{\circ}$ counterclockwise at a distance of about $15^{\prime \prime}(530 \mathrm{pc})$ from the nucleus. At that position a kink in the western $\mathrm{CO}$ ridge has already been observed by Plante et al. (1991) as component 2. It has been interpreted as being due to a deflection of the jet by a dense molecular cloud.

The increase of intensity in the outermost $10^{\prime \prime}$ of our observed area is artificial and due to the increase of the rms-noise 


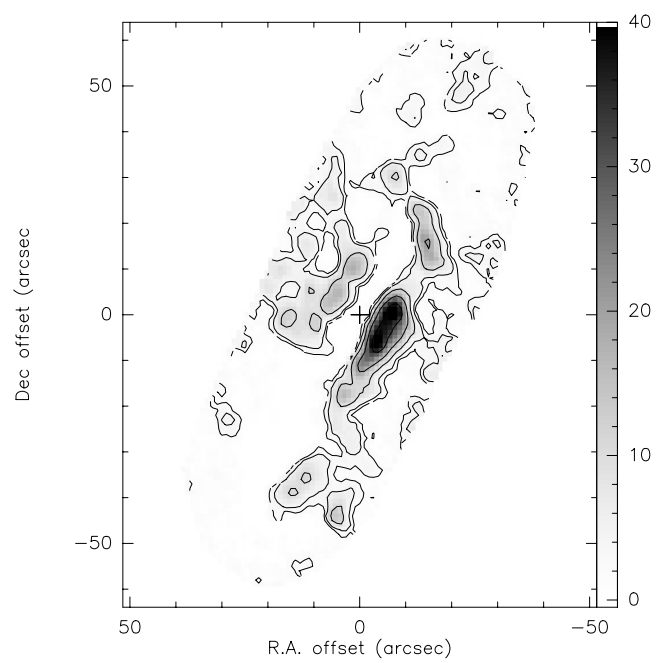

Fig. 2. Integrated intensity of ${ }^{12} \mathrm{CO}(1-0)$ toward the central region of NGC 4258. The rms-noise in the inner part of the map is $0.7 \mathrm{Jy} /$ beam $\mathrm{km} \mathrm{s}^{-1}$. The contour levels are 2, 4, 10, 20, 35, $50 \mathrm{Jy} /$ beam $\mathrm{km} \mathrm{s}^{-1}$. The cross marks the nucleus.

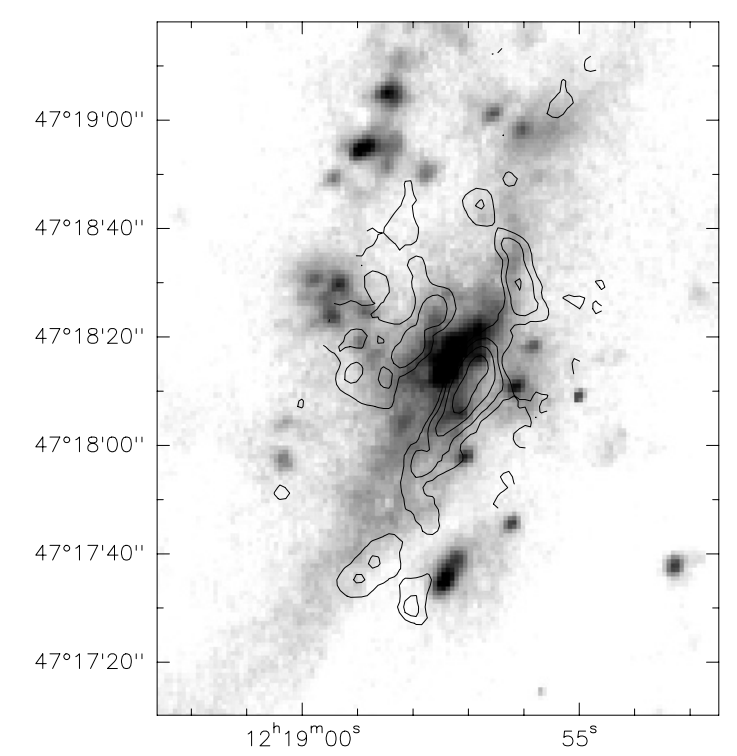

Fig. 3. Contours of the integrated intensity of ${ }^{12} \mathrm{CO}(1-0)$ of NGC 4258 (equal to those in Fig. 2) superimposed onto the $\mathrm{H} \alpha$ image of the galaxy. The normal spiral arms are located in the north and south.

because of the primary beam correction. This effect is most clearly visible along the western edge of the map. The superposition of the $\mathrm{CO}$ distribution on our $\mathrm{H} \alpha$ map is presented in Fig. 3 and shows that the $\mathrm{CO}$ ridges follow the $\mathrm{H} \alpha$ emission.

The $\mathrm{H} \alpha$ emission is more extended than the $\mathrm{CO}$ emission. It has its maximum in between the two CO ridges with a slight shift towards the stronger western ridge. The $\mathrm{H} \alpha$ emission however is broader than the $\mathrm{CO}$ emission and outlines the jet to larger distances from the nucleus. Contrary to previous interpretations of Martin et al. (1989) and Plante et al. (1991, 1994) the CO does not outline "walls" around the $\mathrm{H} \alpha$ jets and hence does not confine it. It rather seems to be mixed in location and in velocity with the $\mathrm{H} \alpha$ gas.

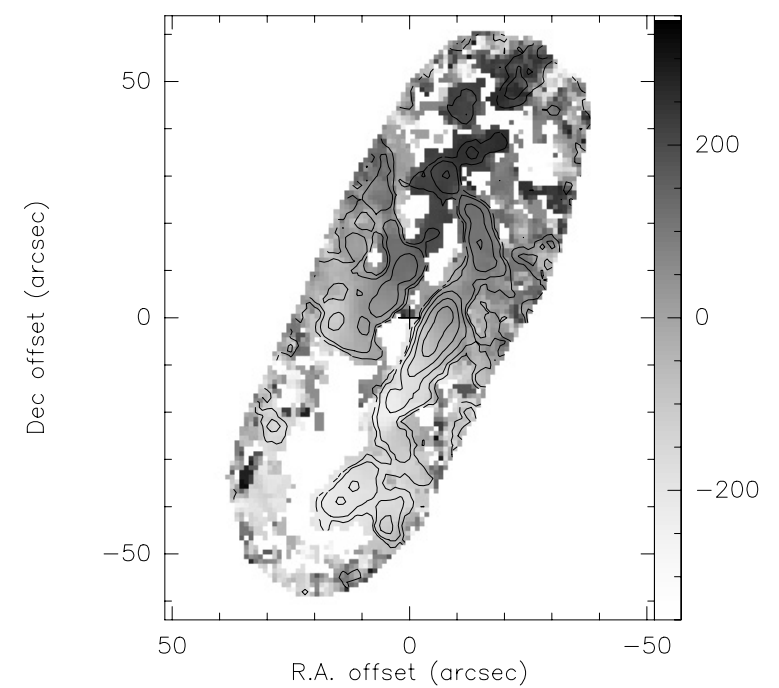

Fig. 4. Iso-velocity map of ${ }^{12} \mathrm{CO}(1-0)$ with respect to the systemic velocity in $\left[\mathrm{km} \mathrm{s}^{-1}\right]$ toward the central region of NGC 4258 (grey scales). All points for which the integrated intensity is below its $3 \sigma$ noise level are discarded. The contours represent the integrated intensity and are equal to those in Fig. 2. The cross marks the nucleus.

\subsection{The velocity distribution}

\subsubsection{Iso-velocity and velocity dispersion maps}

Figure 4 shows the iso-velocity or first moment map of our spectra. The velocities in the southern part of NGC 4258 are blue shifted whereas those in the northern part are red shifted with velocities up to about $200 \mathrm{~km} \mathrm{~s}^{-1}$ (north), resp. up to $-200 \mathrm{~km} \mathrm{~s}^{-1}$ (south). This is indeed expected for normal galactic rotation and indicates that most of the molecular gas takes part in the galactic rotation. A comparison with the rotation curves found in $\mathrm{HI}$ and $\mathrm{H} \alpha$ is given in Sect. 3.3.2. At close look the iso-velocity pattern reveals however a peculiarity: we observe a significant velocity gradient between the eastern and western side of the major axis of the galaxy at the same distance from the nucleus. That is unusual for normal galactic rotation and will be discussed in Sect. 4.3.1.

The velocity dispersion or second moment map is given in Fig. 5. For a better comparison we superimposed also here the contours of the integrated intensity as presented in Fig. 2.

It shows that the velocity dispersion along the ridges is generally large with values of about 80 to $160 \mathrm{~km} \mathrm{~s}^{-1}$ along the western ridge up to a distance of about $25^{\prime \prime}$ from the nucleus. Further outwards the velocity dispersion decreases to values of $\leq 60 \mathrm{~km} \mathrm{~s}^{-1}$. The velocity dispersion seems to reflect the multiple component structure of many spectra just in the inner part whereas the spectra farther away from the nucleus have only one component.

\subsection{2. $p-v$ diagrams along the major axis of the galaxy}

We determined several position velocity diagrams along the major axis of NGC 4258 and parallel to it with a separation of 4 " to a distance of $\pm 20^{\prime \prime}$ from the major axis. The orientation of the major axis deviates only by $5^{\circ}$ from the orientation of the $\mathrm{CO}$ ridges and was found to be a good linear approximation for the position of the jet axis. The principal features of the p-v diagrams are not sensitive to the exact position of the axes chosen. The p-v diagrams are shown in Fig. 6 together with the integratedintensity (as shown in Fig. 2) in the lowest right panel. 


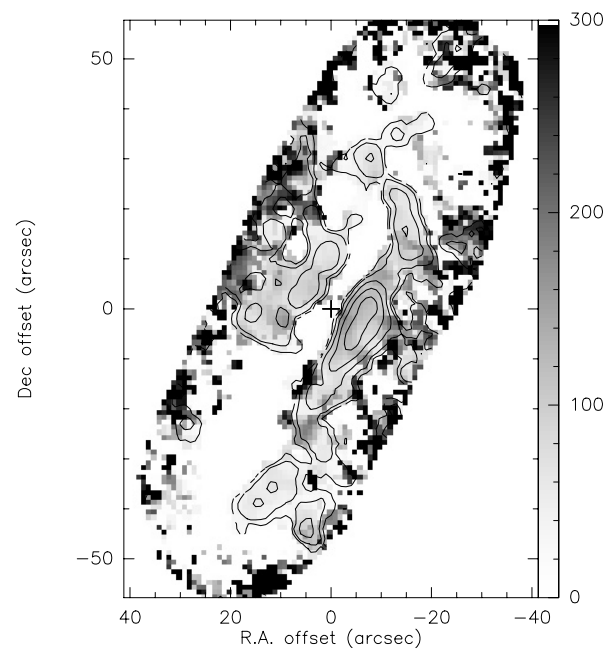

Fig. 5. Map of the velocity dispersion in $\left[\mathrm{km} \mathrm{s}^{-1}\right]$ of ${ }^{12} \mathrm{CO}(1-0)$ toward the central region of NGC 4258 (grey scales). Points for which the integrated intensity is below the $3 \sigma$ noise level have been omitted. The contours represent the integrated intensity and are equal to those in Fig. 2. The cross marks the nucleus.

As expected from the strong $\mathrm{CO}$ depletion between the two ridges in the integrated intensity map, the $\mathrm{p}-\mathrm{v}$ diagram along the major axis (position $0^{\prime \prime}$ ) has only very low intensity. The highest intensities are found at the $\mathrm{p}-\mathrm{v}$ diagrams at positions $+4^{\prime \prime}$, $-8^{\prime \prime}$, and $-12^{\prime \prime}$ east and west along the major axis. The dominant shape and structure is elongated and rises from negative velocities in the south-east to positive velocities in the north-west. Such a feature is close to what is expected for $\mathrm{CO}$ gas rotating in a galactic disk.

A comparison of our $\mathrm{p}-\mathrm{v}$ diagram with the HI rotation curve by van Albada (1980) shows that the CO velocities rise steeper than the HI velocities which reach velocities of about $200 \mathrm{~km} \mathrm{~s}^{-1}$ only at distance of about $40^{\prime \prime}$ from the nucleus and then seem to flatten further out. The angular resolution of the HI observations is however only $30^{\prime \prime}$ along the major axis. This means that we can only see an average of all HI velocities between e.g. $0^{\prime \prime}$ and $30^{\prime \prime}$ from the nucleus. Hence the HI velocities are only lower limits for the true rotation of the ISM in NGC 4258 and the deviation of our $\mathrm{CO}$ velocities from the $\mathrm{HI}$ rotation velocities does not contradict our interpretation of $\mathrm{CO}$ being a rotating molecular disk gas.

Peculiar features in the pv-diagrams are the parallel structures that are visible in some diagrams, mostly in those at $8^{\prime \prime}$ at positive velocities and at $-4^{\prime \prime},-8^{\prime \prime},-12^{\prime \prime}$ at negative velocities. It means that $\mathrm{CO}$ clouds with equal velocities are found at two different distances from the nucleus along the corresponding cut. This is also visible in the iso-velocity map in Fig. 4. The typical distance between the parallel structures in Fig. 6 is about $20^{\prime \prime}$.

\subsection{3. $\mathrm{p}-\mathrm{v}$ diagrams perpendicular to the major axis of the galaxy}

We further determined $\mathrm{p}-\mathrm{v}$ diagrams perpendicular to the major axis (the jet axis), i.e. along the minor axis and parallel to it with a separation of $5^{\prime \prime}$ up to a distance of $40^{\prime \prime}$ to the north and south, respectively. Three characteristic ones are presented in Fig. 7.

Up to a distance of $30^{\prime \prime}$ from the nucleus most of these $p-v$ diagrams perpendicular to the jet axis show two CO blobs shifted to each other by about $15^{\prime \prime}$ in position and about $50 \mathrm{~km} \mathrm{~s}^{-1}$ in velocity. These two blobs (we mean the two strongest components in Fig. 7) move together systematically from negative (blue shifted) velocity to positive (red shifted) velocity when one compares the different $\mathrm{p}-\mathrm{v}$ diagrams from south to north. The eastern blobs are generally more red shifted than the western ones. A close comparison with the p-v diagrams along the jet axis reveals that the blobs of $\mathrm{CO}$ emission show the systematic velocity shift between the 2 CO-ridges as described in Sect. 3.3.1.

\subsection{CO mass estimate and distribution of the $\mathrm{CO}$ emission}

The integrated CO flux in a $600 \mathrm{~km} \mathrm{~s}^{-1}$ wide band within the area of emission (see Fig. 2) is $630 \mathrm{Jy} \mathrm{km} \mathrm{s}^{-1}$, respectively $102 \mathrm{~K} \mathrm{~km} \mathrm{~s}^{-1}$ (the data are corrected for missing spacings and primary beam effects). This corresponds to a $\mathrm{CO}$ luminosity of $2.6 \times 10^{8} \mathrm{~K} \mathrm{~km} \mathrm{~s}^{-1} \mathrm{pc}^{2}$ in agreement with the value found by Krause et al. (1990) and Cox \& Downes (1996) with the Pico Veleta telescope (note however that the assumed distance to NGC 4258 is slightly different in these papers). Assuming the "standard" $N\left(\mathrm{H}_{2}\right) / \mathrm{I}_{\mathrm{CO}}$ conversion factor of $2.3 \times$ $10^{20} \mathrm{~mol} \mathrm{~cm}^{-2}\left(\mathrm{~K} \mathrm{~km} \mathrm{~s}^{-1}\right.$ (Strong et al. 1988) we derive a mass of molecular hydrogen of $M_{\mathrm{H}_{2}} \simeq 10^{9} M_{\odot}$. As already argued in Krause et al. (1990) this mass derived from the conventional $\mathrm{CO} / \mathrm{H}_{2}$ conversion may be an unreliable estimate of the total mass of molecular hydrogen due to possible extreme physical conditions along the jets. It is well in the range of what is expected as total molecular hydrogen content in the inner disk of a spiral galaxy, also those with a Seyfert 2 active nucleus (e.g. Young \& Scoville 1991; Elmouttie et al. 1998).

In NGC 4258 however, the CO emission is only concentrated along the inner jets up to a radial distance of about $2 \mathrm{kpc}$ (Krause et al. 1990). No CO emission further outwards than $2 \mathrm{kpc}$ along the jets could be detected. This was confirmed by further observations of García-Barreto (private communication) who extended the Pico Veleta CO map to the north up to a nuclear distance of $\left.120^{\prime \prime}(3.8 \mathrm{kpc})\right)$. He could also not detect $\mathrm{CO}$ emission along the northern normal spiral arm in NGC 4258.

The two blobs of $\mathrm{CO}$ emission north of the nucleus at a distance of about $50^{\prime \prime}$ that are visible in the CO map of the BIMA survey (Regan et al. 2001) are also visible in the Pico Veleta single dish map (Krause et al. 1990) but not within the area that was covered by our present PdB interferometer map. From the Pico Veleta map alone it was not really clear whether they belong to the northern spiral arm or the northern jet. The BIMA survey shows their (projected) position in between the normal spiral arm and the northern jet, their velocities (shown in Helfer et al. 2003), however, deviate strongly from those expected for the normal spiral arm at this nuclear distance. Hence we conclude that it cannot be regarded as a $\mathrm{CO}$ detection in the normal spiral arm in NGC 4258.

\section{Discussion}

\subsection{CO as jet tracer}

The main observed $\mathrm{CO}$ features, i.e. the concentration of $\mathrm{CO}$ in two ridges along the jet axis as well as the lack of $\mathrm{CO}$ in other parts of the galaxy may possibly be explained by an interaction process of the jet with the interstellar medium.

Cox \& Downes (1996) proposed that the elongated CO feature traces a bar flow in NGC 4258 rather than being associated with the nuclear jet. An HI bar has indeed been found in this galaxy (van Albada 1980) but at a position angle deviating by $35^{\circ}$ from the observed $\mathrm{CO}$ structure (see Table 1). Our high resolution observation now reveals that the $\mathrm{CO}$ has 


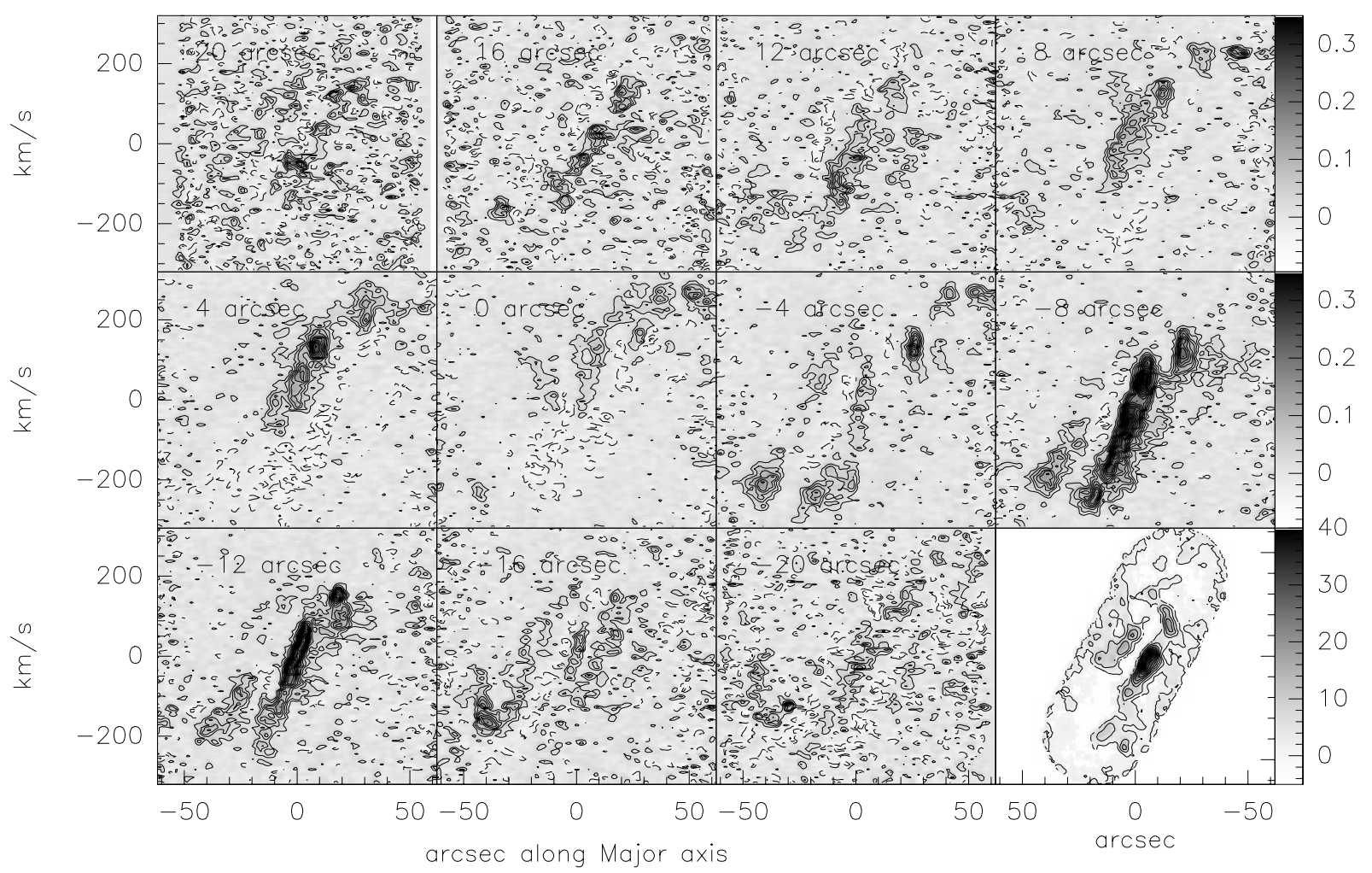

Fig. 6. Position-velocity diagram of NGC 4258 along the CO-ridges and parallel to it with distances from $20^{\prime \prime}$ east (upper left panel) to $20^{\prime \prime}$ west (panel labeled with -20 arcsec). The relative positions are as in Fig. 2: $-50^{\prime \prime}$ along the major axis means southeast and 50" northwest of the nucleus. The intensities are given in grey scale, their units are given by the wedges in $\left[\mathrm{Jy} / \mathrm{beam} \mathrm{km} \mathrm{s}{ }^{-1}\right]$. The lower right panel shows again the integrated intensity (as shown in Fig. 2) for comparison.

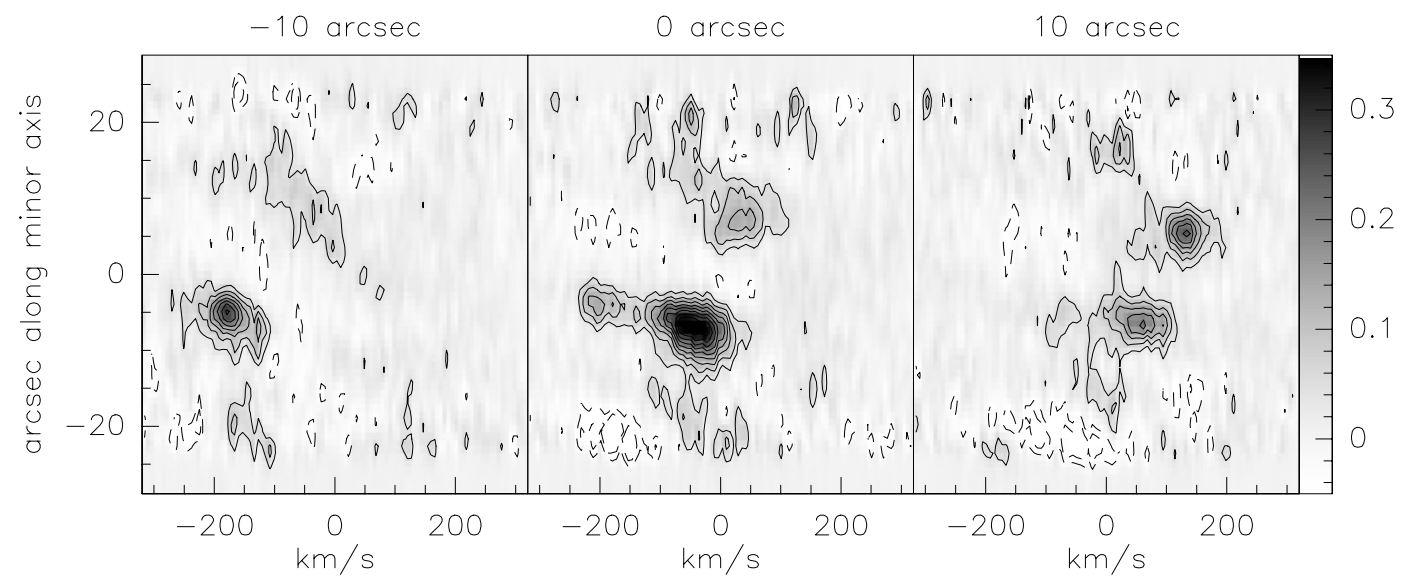

Fig. 7. Position-velocity diagrams of NGC 4258 perpendicular to the Co-ridges at distances $-10^{\prime \prime}$ (south), $0^{\prime \prime}$, and $10^{\prime \prime}$ (north) from the nucleus. As in Fig. 2, 10" along the minor axis means northeast and $-10^{\prime \prime}$ southwest of the nucleus.

a funnel in between two parallel ridges. The appearance of our p-v diagrams (Fig. 6) has not the rhomboid structure typical for bars (e.g. García-Burillo \& Guélin 1995). Hence we exclude the bar hypothesis (which has already been rejected for other reasons by Hyman et al. 2001; Sawada-Satoh et al. 2007) and assume that the $\mathrm{CO}$ structure is related to the jets.

The high amount of molecular gas observed along the jets and the lack of CO elsewhere in the disk of NGC 4258 already suggest that the molecular gas was not ejected from the nucleus itself but originates from galactic disk gas which has interacted with the jets. This implies that the kpc-scale jet must be located more or less within the galactic disk (see also Daigle \& Roy 2001). The typical scale-height of the CO layer in galactic disks constrains the deviation of the kpc-scale jet axis from the galactic plane to angles between $1^{\circ} .5$ and $3^{\circ}$ (Krause et al. 1990).

\subsection{The jet orientation}

We now discuss observational constraints on the geometry of the jet orientation.

The jet itself as seen in the radio emission shows a kink of $40^{\circ}$ at a radial distance of $24^{\prime \prime}(850 \mathrm{pc})$ symmetrically on both sides of the center (see Table 1). The position angle of the $\mathrm{H} \alpha$ and the $\mathrm{CO}$ feature is just the mean jet direction in the radio continuum before and behind this kink. The $\mathrm{H} \alpha$ emission has 
always been interpreted as being related to the jet (e.g. Cecil et al. 1992).

The position angle (PA) of the radio jet before the kink $\left(r<24^{\prime \prime}\right)$ is along the present spin axis of the accretion disk. Also a VLBA radio jet on sub-pc-scale has been observed along this direction (Herrnstein et al. 1998a; Cecil et al. 2000). Pringle et al. (1999), determined the angle $\beta$ between the nuclear jet axis and the rotational axis of the galaxy applying

$\cos \beta=\sin \delta \sin i \sin \phi+\cos i \cos \phi$,

with the inclination of the galaxy $i$, the inclination of the inner nuclear accretion disk $\phi$ (supposed to be perpendicular to the nuclear jet), and the angle $\delta$ between the position angle of the major axis and that of the projected nuclear jet direction (see Table 1).

Assuming $i=72^{\circ}, \phi \simeq 82^{\circ}$ (Herrnstein et al. 1999), and $\delta=27^{\circ}$, we derive $\beta=62^{\circ}$ (similar to the values given by Pringle et al. 1999 or Wilson et al. 2001). Minor deviations from these values exist in the literature. For example, Wilson et al. (2001) discuss a disk inclination of $i=64^{\circ}$. A recent paper by Herrnstein et al. (2005) apply model fits of the maser data to a warped disk model, indicating warps in inclination as well as in position angle. The inclination warp of the inner accretion disk derived there follows a $i=0.28+0.34 z$ distribution where $i$ is measured in radians and $z$ in milliarcseconds along the l.o.s. This results in a disk inclination of $\phi \simeq 74^{\circ}$ close to the center (i.e. for $z=0$; see caption Fig. 10 or Eq. (14) for $r_{i}=0$ in Herrnstein et al. 2005 ${ }^{1}$ ). Applying Eq. (1) for such a value will result in an offset angle $\beta=60^{\circ}$. The difference to the value derived above is negligible in spite of the rather large offset in the nuclear jet direction of about $8^{\circ}=(82-74)^{\circ}$.

In addition to the $60^{\circ}$-offset in alignment between jet axis and rotation axis of the galaxy, it is worthwhile to note that the spin vectors of nuclear disk and galactic disk are actually antiparallel, i.e. the offset between the spin vectors is $120^{\circ}$ and not $60^{\circ}$. This can easily be seen by comparing the Keplerian rotation of the nuclear disk (e.g. Herrnstein et al. 1999) with e.g. our CO data (Fig. 4).

As the jet inclination angle is close to $90^{\circ}$ the knowledge of the exact jet orientation would be essential if we would derive e.g. the velocity structure of the nuclear jet due to the $1 / \cos (i)$ effect. However, we do not calculate and do not need to know the velocity of the nuclear jet, actually there are no velocity data for the nuclear jet available. In the next section we will discuss velocities of the kpc-jet and compare them with our model scenario. This is independent of the nuclear jet orientation.

For the outer radio jet with $\delta=13^{\circ}$ we derive $\beta=75^{\circ}\left(73^{\circ}\right)$ for $\phi=82^{\circ}\left(74^{\circ}\right)$. For the position angle of the $\mathrm{H} \alpha$ jet (and the $\mathrm{CO}$ feature) we get $\delta=5^{\circ}$ and an angle between the $\mathrm{H} \alpha$ jet and the rotational axis of the galaxy $\beta=83^{\circ}\left(80^{\circ}\right)$ for $\phi=82^{\circ}$ $\left(74^{\circ}\right)$. (The values for $\beta$ with $\phi=74^{\circ}$ i.e. the warped disk model (Herrnstein et al. 2005) are also summarized in Table 1.) This is in rough agreement with the conclusion made above that the $\mathrm{H} \alpha$ jet and the $\mathrm{CO}$ feature must lie within the galactic disk. Again, we point out that the PA of the radio jets before and after the kink are just smaller and larger than the PA of the $\mathrm{H} \alpha$ jet and the $\mathrm{CO}$ feature.

The cause for such an apparent change in the direction of jet propagation is, however, unclear. We note that kinks and apparent changes in the jet flow direction are common among

\footnotetext{
1 Note that the authors plot the disk warping across the very center of the disk, however, the jet direction as indicated is not perpendicular to the disk.
}

extragalactic jets (e.g. Parma et al. 1987). Also, the NGC 4258 jet tracers $\mathrm{CO}$ and $\mathrm{H} \alpha$ show clear evidence for inhomogeneities of the interstellar medium and deflections of the straight jet propagation.

However, note that the change of direction between inner and outer radio jet is symmetric for the jet and counter jet indicating on a systematic mechanism working symmetrically on both sides of the jet. Precession of the jet may be one of the possible mechanisms. There are two indicators for ongoing precession observed. One is the fact that the nuclear accretion disk is inclined towards the equatorial plane of the galaxy (Miyoshi et al. 1995). The other is the observed warping of the nuclear accretion disk (Herrnstein et al. 1996, 2005).

If precession is really in action, the nuclear jet would precede along a cone intersecting the equatorial plane of the galaxy at a certain time. It would interact with the galactic disk just during the time of intersection and the features of interaction (like $\mathrm{H} \alpha$ and $\mathrm{CO}$ ) would appear at those position angles that belong to $\beta \simeq 90^{\circ}$, which gives a $\mathrm{PA} \simeq-28^{\circ}$ (from Eq. (1)) of the observed features. This is within the errors similar to the observed PA of the $\mathrm{H} \alpha$ and $\mathrm{CO}$ features. Therefore, the funnel observed in $\mathrm{CO}$ may also be caused by the jet propagation during earlier times and probably with different strength.

For the following we will assume that the $\mathrm{H} \alpha$ and $\mathrm{CO}$ features are related to the jets in NGC 4258 and we will refer to their direction (PA) as "kpc-scale jet" direction. If we further assume that the kpc-scale jet lies within the galactic disk (i.e. $\beta \simeq 90^{\circ}$ ), the inclination $i_{\text {jet }}$ of the jet towards the line of sight is

$\tan i_{\text {jet }}=(\sin \delta \tan i)^{-1}$

With $\delta=5^{\circ}$ and $i=72^{\circ}$ it follows $i_{\text {jet }}=75^{\circ}$. This means that the northern jet points towards the observer (and the southern jet away from the observer) as its position angle is west of the northern major axis of NGC 4258. However, a jet inclination of $75^{\circ}$ also means that the jet lies close to the plane of the sky. As we observe strong kinks in the jet at $d= \pm 24^{\prime \prime}$ in the position angle we also have to include the possibility of kinks in the direction along the line of sight. Such a kink (which is not detectable in our line observations and maybe also hidden in the $\mathrm{H} \alpha$ observations) could lead to a (projected) geometry where the jets may be pointing away from us in the northern half (and towards us in the southern half). The two different geometric models lead, however, to different interpretations of the observed velocities in the line observations and will be discussed in the next section.

\subsection{Jet propagation and $\mathrm{CO}$ ridges}

We will now discuss both geometric scenarios and their implications on the question how molecular gas can have been accumulated along the kpc-scale jet funnel.

In the first scenario (i) the angle between the jet axis and the plane of the sky varies from a small positive (negative) value to a small negative (positive) value. The northern jet axis points away from the observer. The observed $\mathrm{CO}$ represents molecular gas entrained by the jet. The jet accelerates the molecular gas observed in CO leading to a linearly increasing velocity. This kind of scenario has its similarities in molecular outflows driven by protostellar jets. As it will be discussed in Sect. 4.3.1 this scenario has to be rejected as an interpretation of the observed $\mathrm{CO}$ distribution and velocities.

In the second scenario (ii) the jet inclination along the jet remains constant and is along the galactic disk of NGC 4258. The northern jet axis points towards the observer. The observed $\mathrm{CO}$ 
represents molecular gas in galactic rotation. The concentration of $\mathrm{CO}$ just along the jet ridges is interpreted as accumulation due to interaction of the rotating gas clouds with the jet's magnetic field. This scenario (that had first been sketched in Krause et al. 2005) will be discussed in Sects. 4.3.2 to 4.3.4 and seems to be able to put the puzzle of observational results into one consistent picture.

\subsubsection{Scenario (i): a jet-driven molecular CO-flow?}

Observations of protostellar outflows showed that large-scale mass entrainment and acceleration of this mass by a highvelocity jet may lead to a linear increase in the velocity of the molecular outflow (Stahler 1994; Lada \& Fich 1996). Timedependent magnetohydrodynamic simulations of jet formation (e.g. Ouyed \& Pudritz 1997; Fendt \& Cemeljic 2002) found a similar increase of jet velocity with distance from the jet source. In our case, in NGC 4258 the velocity distribution observed along the $\mathrm{CO}$ ridges shows as well a linear increase with distance from the center. If we interpret these data in analogy to the protostellar outflows, the linear increase seen in the $\mathrm{CO}$ velocity would correspond to that of a continuously accelerated jet-driven molecular flow. We denote this model scenario with (i) and discuss its validity in the following.

We first estimate the kinematic properties of the jet and the potential molecular flow. The jet mass flow rate is not known from observations. In general, observations of astrophysical jets as well as theoretical arguments (e.g. Ferreira 1997) showed that typically $1 \%-10 \%$ of the disk accretion rate becomes ejected into the jets. In the case of NGC 4258, various number values for the nuclear accretion rate were published so far. Gammie et al. (1999) obtained $\dot{M}_{\mathrm{a}} \geq 1.5 \times 10^{-4} M_{\odot} \mathrm{yr}^{-1}$ for a thin warped disk, and $\dot{M}_{\mathrm{a}} \simeq 10^{-2} M_{\odot} \mathrm{yr}^{-1}$ for an advection-dominated disk. A standard accretion disk model gives $\dot{M}_{\mathrm{a}} \simeq 7 \times 10^{-5} \alpha M_{\odot} \mathrm{yr}^{-1}$ (Neufeld \& Maloney 1995) with a standard accretion disk viscosity parameter $\alpha$. Both alternatives, the advection dominated disk and the standard accretion disk model seem to be consistent with the observations (Herrnstein et al. 1998b). Yuan et al. (2002) explained certain spectral properties by shock emission from the jet basis, thus lowering the nuclear jet mass loss rate to about $\dot{M}_{\mathrm{j}} \simeq 3 \times 10^{-4} M_{\odot} \mathrm{yr}^{-1}$. Recent observations by Modjaz et al. (2005) revealed similarly low accretion rates indicating an upper limit of $\dot{M}_{\mathrm{j}} \simeq 10^{-3.7} M_{\odot} \mathrm{yr}^{-1}$.

In the following we adopt a nuclear accretion rate of $\dot{M}_{\mathrm{a}} \leq$ $10^{-4} M_{\odot} \mathrm{yr}^{-1}$ corresponding to an upper limit for the nuclear jet mass flow rate $\dot{M}_{\mathrm{j}} \leq 10^{-5} M_{\odot} \mathrm{yr}^{-1}$. With that we estimate the kinetic power of the nuclear jet (i.e. the jet nearby to the accretion disk) as

$L_{\mathrm{j}} \simeq 6 \times 10^{39} \mathrm{erg} \mathrm{s}^{-1}\left(\frac{v_{\text {jet }}}{0.1 c}\right)^{2}\left(\frac{\dot{M}_{\mathrm{j}}}{0.1 \dot{M}_{\mathrm{a}}}\right)\left(\frac{\dot{M}_{\mathrm{a}}}{10^{-4} M_{\odot} \mathrm{yr}^{-1}}\right)$.

This value is uncertain as both the nuclear jet velocity and mass loss rate in NGC 4258 are unknown. Cecil et al. (1995b) discussed a nuclear jet velocity of $0.1 c$. However, there are frequent cases where nuclear jets in AGN propagate with velocities close to the speed of light. With a nuclear jet velocity of $0.9 c$ the kinetic luminosity increases to about $L_{\mathrm{j}} \simeq 5 \times 10^{41} \mathrm{erg} / \mathrm{s}$, a value which is similar to the nuclear jet radio power derived by Falcke \& Biermann (1999). Clearly, if the nuclear jet kinetic power would be entirely tranformed into radio emission, no energy available to drive the kpc-scale jet seen in $\mathrm{H} \alpha$ or $\mathrm{CO}$. In this respect, our estimate for the kinetic power represents a lower limit.
An essential property for our discussion below is the kinematic time scale of the kpc-scale jet (i.e. the jet at distance of the order of $\mathrm{kpc}$ ) which has to be compared to the typical time scale of other processes possibly involved. The kinematic time scale can be derived from the jet characteristic length and velocity, but (as already indicated) the jet speed is not really known. Several approaches to estimate the jet velocity have been discussed in the literature. Cecil et al. (1992) considered $\mathrm{H} \alpha$ data and derived a ballistic jet velocity about $2000 \mathrm{~km} \mathrm{~s}^{-1}$ for a model of "braided" jet "strands" ejected from orbiting nuclear sources. However, such a scenario seems to be ruled out by the observations of the nuclear jet and the surrounding masering disk. The hot ionized gas observed in the X-ray band indicates shock heating with inferred shock velocities of about $350-500 \mathrm{~km} \mathrm{~s}^{-1}$ (Cecil et al. 1995a,b). In general, the actual jet velocity depends on the physical (hydrodynamic) conditions of the shock environment and is typically several times larger. Daigle \& Roy (2001) investigated possible jet trajectories across the galactic disk discussing a possible velocity range between 500 and $10^{4} \mathrm{~km} \mathrm{~s}^{-1}$. They finally applied $4000 \mathrm{~km} \mathrm{~s}^{-1}$ as $\mathrm{kpc}$-scale jet velocity in NGC 4258. Eventually, we estimate the kinematic time scale of the kpc-scale jet by considering the extension of the $\mathrm{H} \alpha$ emission of $l_{\mathrm{H} \alpha} \simeq 12 \mathrm{kpc}$ (see Cecil et al. 2000) and a jet velocity derived from the $\mathrm{H} \alpha$ emission $v_{\mathrm{H} \alpha} \simeq 2000 \mathrm{~km} \mathrm{~s}^{-1}$ as a representative value discussed in the literature,

$\tau_{\mathrm{kin}} \equiv \frac{l_{\mathrm{H} \alpha}}{v_{\mathrm{H} \alpha}} \simeq 6 \times 10^{6} \mathrm{yrs}\left(\frac{12 \mathrm{kpc}}{l_{\mathrm{H} \alpha}}\right)\left(\frac{2000 \mathrm{~km} \mathrm{~s}^{-1}}{v_{\mathrm{H} \alpha}}\right)^{-1}$.

Note, that the fact that the "anomalous arms" (radio) in NGC 4258 have an extent larger than the whole galaxy (Hummel et al. 1989) may indicate that the nuclear "jet activity" has been present for a considerably longer period, and the corresponding kinematic time scale and the amount of mass injected into the jet from the nuclear accretion disk could be appropriately larger.

Within the kinematic time scale of $6 \times 10^{6} \mathrm{yr}$ a total mass of about $10^{2} M_{\odot}$ can be accumulated by the nuclear jet in the case of a mass loss rate of $\dot{M}_{\mathrm{j}} \simeq 10^{-5} M_{\odot} \mathrm{yr}^{-1}$. This is in any case far below the value of the accelerated mass inferred from the $\mathrm{H} \alpha$ observations $\left(1.5 \times 10^{6} M_{\odot}\right.$, Cecil et al. 1992) and CO observations $\left(10^{9} M_{\odot}\right.$, this paper). Hence, one may conclude that most of the material observed in $\mathrm{H} \alpha$ and $\mathrm{CO}$ cannot be launched at the jet source but could only be entrained from the interstellar medium.

If we further follow the model assumption in this section and interpret the $\mathrm{CO}$ velocity distribution observed in NGC 4258 as linear velocity of a molecular flow assuming an inclination of that flow of $i_{\text {jet }} \simeq 75^{\circ}$ as derived in Eq. (2), the de-projected molecular flow velocity is $\left(200 \mathrm{~km} \mathrm{~s}^{-1} / \cos 75^{\circ}\right) \simeq 800 \mathrm{~km} \mathrm{~s}^{-1}$. This would correspond to the velocity of the gas entrained by the jet and would be in rough agreement (within a factor of two) with the jet velocity estimated above (but is of course highly uncertain due to the high inclination). Therefore, from the interpretation of the velocity information we have, the model of an entrained molecular flow would be feasible.

However, it would be difficult to understand how this amount of molecular mass could actually have been accelerated by a jet with parameters as estimated for the NGC 4258 nuclear jet as there is obviously a strong mismatch between the kinetic momentum of both components. The kinetic momentum of the nuclear jet $P_{\text {jet }} \simeq 3 \times 10^{2} M_{\odot} \cdot 0.1 \mathrm{c}=2 \times 10^{45} \mathrm{~g} \mathrm{~cm} \mathrm{~s}^{-1}$ is much lower compared to what is observed for the kpc-scale molecular flow in $\mathrm{CO}$ or $\mathrm{H} \alpha$ (assuming that the observed velocity distribution refers to a linear motion). Unless we assume much higher jet 


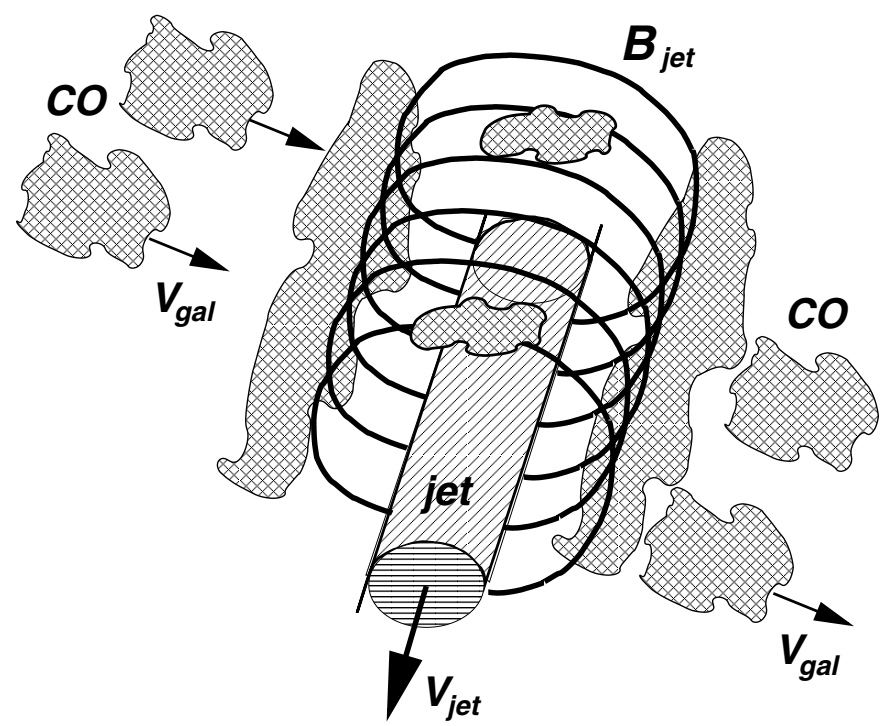

Fig. 8. Model scenario (ii) of the jet in NGC 4258. The highly collimated jet stream carries a net poloidal current equivalent to a surrounding toroidal magnetic field. $\mathrm{CO}$ clouds in galactic rotation interact with the jet's toroidal magnetic field. Most of the CO continues with the galactic rotation after being slightly decelerated across the jet.

mass flow rates of the nuclear jet in the past (probably triggered by a higher accretion rate) or a higher jet velocity (e.g. $>0.9 c$ ), we must dismiss this scenario of a jet-driven molecular flow as proposed previously in this section. This holds even more as this scenario is unable to explain some other observational results like the total $\mathrm{CO}$ intensity distribution and the detailed structure in the $\mathrm{p}-\mathrm{v}$ diagrams.

\subsubsection{Scenario (ii): a current carrying jet capturing interstellar CO}

If we consider model scenario (ii) to explain our observations, the observed $\mathrm{CO}$ can be interpreted as molecular disk gas in galactic rotation. Its rotational velocity rises strongly in the central region up to a distance of about $8^{\prime \prime}(280 \mathrm{pc})$ and seemingly flattens further outwards.

The p-v curve along the major axis (see Fig. 6) is steeper than the rotation curve observed in HI (van Albada 1980) but not as steep and irregular as the velocities observed in $\mathrm{H} \alpha$ by Dettmar $\&$ Koribalski (1990) and Cecil et al. (1992). A steeper rise of the velocities in the central part of a galaxy in $\mathrm{CO}$ compared to HI has already been observed in many galaxies (e.g. Sofue 1997) and is rather expected due to the very different angular resolutions in both maps as discussed in Sect. 3.3.2. Hence the $\mathrm{CO}$ in the disk of NGC 4258 is to first order in "normal" galactic rotation.

However, the kinematics of the molecular gas are also affected by the kpc-scale jets. The non-detection of $\mathrm{CO}$ outside the region along the inner jets can be explained by an interaction of the molecular gas with the jets. The $\mathrm{CO}$ clouds become temporarily "captured" leading to a longer staying time of the gas near to the jets than elsewhere (see Fig. 8). We suggest that the reason for this interaction is a toroidal magnetic field of the current-carrying jets (an interaction process being somewhat similar to the formation of spiral arms due to a perturbation of the gravitational potential).

This jet-cloud interaction hypothesis is supported by the distinct velocity and spatial structure of the observed $\mathrm{CO}$ which becomes obvious in Fig. 7. The gap in the CO intensity between the two blobs in the $\mathrm{p}$-v diagrams perpendicular to the major axis can be interpreted as the central funnel of the jets which is depleted of molecular gas and indeed follows the gap between the two ridges in Fig. 2.

The relative velocity shift between the two blobs is approximately constant along the jet axis. It is such that the $\mathrm{CO}$ velocities (absolute values) on the upstream side (with respect to the rotation of the disk gas) of the jets are smaller than those on the downstream side. This and also the double structure in Fig. 6 clearly indicates that interaction between the molecular disk gas and the jets is present and influences the rotation of the molecular gas.

The observation of $\mathrm{H} \alpha$ fits into our picture as signature of former molecular gas which has been shock-ionized during the partly violent interaction processes with the jets (as already proposed by Cecil et al. 1992).

Having given the motivation for our model hypothesis of a magnetic jet-cloud interaction in NGC 4258, a more detailed discussion requires estimates of various physical parameters such as kinetic power, magnetic field strength, and corresponding time scales.

\subsubsection{The jet magnetic field}

Polarimetric VLA observations of water vapor maser emission close to the central engine of NGC 4258 gave an upper limit for the parallel component of the nuclear disk magnetic field (i.e. the toroidal field) at a disk radius of $0.2 \mathrm{pc}$ of about $90 \mathrm{mG}$ (Modjaz et al. 2005). Note that its distance corresponds to about 50000 Schwarzschild radii from the central black hole and is certainly not identical to the origin of the jet which is expected to be as close to the black hole as about 10-50 Schwarzschild radii.

Nevertheless, theoretical models of magnetized jets suggest that the disk electric current $I_{\text {disk }} \propto r_{\text {disk }} B_{\phi \text {,disk }}$ is conserved. We may therefore take the observed toroidal field strength at $0.2 \mathrm{pc}$ and extrapolate it to the inner (unresolved) disk region of jet formation

$I_{\mathrm{disk}} \simeq$ const $=3 \times 10^{17} \mathrm{~A}\left(\frac{r}{0.2 \mathrm{pc}}\right)\left(\frac{B_{\phi, \mathrm{disk}}}{100 \mathrm{mG}}\right)$.

Assuming that the electric current in the disk-jet system is roughly conserved, $I_{\text {jet }} \simeq I_{\text {disk}}$, we estimate the kpc-scale jet toroidal magnetic field as

$$
\begin{aligned}
B_{\phi, \text { jet }} & \leq 0.2 \mathrm{mG}\left(\frac{r_{\text {jet }}}{100 \mathrm{pc}}\right)^{-1}\left(\frac{I_{\text {jet }}}{3 \times 10^{17} \mathrm{~A}}\right) \\
& =0.2 \mathrm{mG}\left(\frac{r_{\text {disk }} / r_{\text {jet }}}{0.002}\right)\left(\frac{B_{\phi, \text { disk }}}{100 \mathrm{mG}}\right),
\end{aligned}
$$

where here $r_{\text {jet }}$ represents a measure for the kpc-scale jet radius. This is consistent with accretion disk energy equipartition arguments suggesting a poloidal current of about $10^{18} \mathrm{~A}$ for active galactic nuclei accretion disks (e.g. Camenzind 1990; Blandford et al. 1990).

At larger distances from the nucleus radio observations detected a large scale magnetic field along the anomalous arms (van Albada \& van der Hulst 1982; Krause et al. 1984; Hummel et al. 1989). Recent radio continuum observations revealed a total magnetic field strength of about $300 \mu \mathrm{G}$ in the northern jet at about $5 \mathrm{kpc}$ distance from the nucleus (with a linear resolution of about 100 pc) (Krause \& Löhr 2004). This value is much 
higher than the usual field strengths measured in spiral galaxies $(1-50 \mu \mathrm{G})$, but agrees surprisingly well with the above estimate in Eq. (6). Note that the above mentioned argument of electric current conservation naturally links the observationally detected field strength in the nuclear disk and the kpc-jet. In practice, dissipative processes will be present in the jet and in the jet-ambient medium interaction layer which will decrease the jet electric current.

\subsubsection{Molecular clouds - time scales and ambipolar diffusion}

Molecular clouds are poorly ionized with a typical degree of ionization of $10^{-7}$ (Pudritz 1990; Shu 1992), and ambipolar diffusion plays an important role for the magnetic field interaction of such clouds. Ambipolar diffusion considers the drift between the ionized and the neutral component of the gas where both are coupled by dynamical friction due to collisions and hence may affect the orbital motion of the molecular clouds if they cross the jet magnetic field. Such an interaction may decelerate the molecular clouds and therefore enhances the probability of finding them along the jet's edges, essentially forming a molecular gas-depleted jet funnel (see Sect. 4.4).

We now estimate the kinematic time scale for the jet-cloud interaction from the travel time of a molecular cloud across the jet assuming a width of the jet funnel ${ }^{2}$ of $2 r_{\text {jet }}$.

The dynamical time scale for a magnetized molecular cloud is given by $\tau_{\mathrm{dyn}}=l_{\mathrm{cl}} / v_{\mathrm{alf}}$, where $l_{\mathrm{cl}}$ is the typical size of the cloud and $v_{\text {alf }}=B / \sqrt{4 \pi \rho}$ denotes the local Alfvén speed. The time scale for ambipolar diffusion - the diffusion of the magnetic field relative to the neutral particles under the influence of collisions with ionized particles - is $\tau_{\mathrm{ad}}=l_{\mathrm{cl}} / v_{\mathrm{d}}$ where $v_{\mathrm{d}}=v_{\mathrm{alf}}^{2} /\left(\sqrt{\rho} l_{\mathrm{cl}} C \gamma_{\mathrm{ad}}\right)$ is the drift velocity with ${ }^{3}$ the drag coefficient $\gamma_{\text {ad }}$ and a constant $C$.

Assuming that a jet magnetic field of strength of $200 \mu \mathrm{G}$ (Eq. (6)) has penetrated the molecular cloud and considering molecular hydrogen $\rho_{\text {cloud }}=2 n_{\mathrm{cl}} m_{\mathrm{H}}$, we obtain

$$
\begin{aligned}
\frac{\tau_{\mathrm{ad}}}{\tau_{\mathrm{dyn}}} & =\sqrt{4 \pi} \frac{\gamma_{\mathrm{ad}} C l \rho}{B} \\
& \simeq 20\left(\frac{l_{\mathrm{cl}}}{10 \mathrm{pc}}\right)\left(\frac{n_{\mathrm{cl}}}{10^{3} \mathrm{~cm}^{-3}}\right)\left(\frac{B_{\text {jet }}}{200 \mu \mathrm{G}}\right)^{-1},
\end{aligned}
$$

where $n_{\mathrm{cl}}$ is the hydrogen particle density. This corresponds to a drift velocity of

$v_{\mathrm{d}}=0.5 \mathrm{~km} \mathrm{~s}^{-1}\left(\frac{B_{\mathrm{jet}}}{200 \mu \mathrm{G}}\right)^{2}\left(\frac{n_{\mathrm{cl}}}{10^{3} \mathrm{~cm}^{-3}}\right)^{-\frac{3}{2}}\left(\frac{l_{\mathrm{cl}}}{10 \mathrm{pc}}\right)$

which is clearly below the mean galactic rotational velocity of the molecular clouds.

In summary, our rough estimate indicates an order of magnitude difference in the time scales $\tau_{\text {ad }} \simeq 20 \tau_{\text {dyn }}$ suggesting that the scenario of an ambipolar jet magnetic field-cloud interaction is feasible. If the dynamical time scale had been much larger than the ambipolar diffusion time scale the (neutral) matter would hardly be affected by the magnetic field. The other extreme case would correspond to a "frozen-in" scenario of field and matter.

\footnotetext{
${ }^{2}$ Here, $r_{\text {jet }}$ is not associated with the actual radius of the jet mass flow but is a measure of the scale length (width) of the jet toroidal magnetic field.

${ }^{3}$ See e.g. Shu (1992) for numerical values of the parameter $\gamma_{\mathrm{ad}}=$ $3.5 \times 10^{13} \mathrm{~cm}^{3} \mathrm{~g}^{-1} \mathrm{~s}^{-1}$ and $C=3 \times 10^{-16} \mathrm{~cm}^{-3 / 2} \mathrm{~g}^{1 / 2}$.
}

\subsection{Interaction of molecular clouds with the jet magnetic field}

In the previous sections we have discussed important estimates of our model scenario (ii) (Fig. 8) which can explain both, the observational results of the molecular $\mathrm{CO}$ being concentrated only along the edges of the kpc jet of NGC 4258, and also the lack of CO elsewhere in the galactic disk of NGC 4258: the molecular gas in galactic rotation interacts with the jet's toroidal magnetic field due to ambipolar diffusion. As stated above, we suggest that by this process the galactic molecular gas becomes partly "captured" by the magnetic field resulting in an enhanced probability of finding the $\mathrm{CO}$ clouds along the jet ridges. Part of the cloud material diffuses across the jet's toroidal magnetic field and will finally hit the jet flow itself. This matter will become shocked, the molecules will become dissociated, and the gas may be detected in $\mathrm{H} \alpha$ across in the $\mathrm{CO}$ depleted funnel. We note that also the apparent velocity change of the clumpy CO components upstream and downstream of the jet (see Fig. 7) suggests such an interaction. As described in Sect. 3.3.3 the velocity of the upstream CO is about $50 \mathrm{~km} \mathrm{~s}^{-1}$ lower than that of the downstream CO.

Such a velocity change is in both, its amount and direction with respect to the galactic rotation, is comparable with the effect on rotating gas in a trailing spiral density wave. The streaming motions by density waves in the plane of the galaxy with respect to purely circular motion has been found to be several $\mathrm{km} \mathrm{s}^{-1}$ up to $70 \mathrm{~km} \mathrm{~s}^{-1}$ as observed for molecular clouds in M 51 (García-Burillo et al. 1993). In NGC 4258 we may observe similar effects on the $\mathrm{CO}$ velocities along both edges of the inner jet and we see a concentration (a "traffic jam") of CO along the jets. This scenario is especially feasible if the jet axis is slightly off the galactic plane. Then the motion of the galactic molecular clouds could only be influenced by the jet's toroidal magnetic field, which extents to larger radii than the jet itself.

The detailed process of interaction is very difficult to investigate, as a number of important parameters are not really known as the magnetic field strength and orientation, the mass flow rate and ionization fraction in the cloud. Numerical magnetohydrodynamic simulations of the jet-cloud interaction may finally prove or disprove our hypothesis. A first check of our model suggestion of a jet-cloud interaction, however, is to compare the energies involved in the jet and cloud motion as these parameters should be in reasonable proportions. The ratio of the kinetic energy density of a molecular cloud to the jet magnetic field energy density is

$$
\frac{E_{\text {kin,cloud }}}{E_{B_{\text {jet }}}}=0.006 n_{\mathrm{cl}}\left(\frac{v_{\text {rot }}}{200 \mathrm{~km} \mathrm{~s}^{-1}}\right)^{2}\left(\frac{B_{\text {jet }}}{200 \mu \mathrm{G}}\right)^{-2} \text {, }
$$

where $n_{\mathrm{cl}}$ is the molecular hydrogen particle density. The cloud on its orbital motion will compress the field until equilibrium is reached. For small densities $\left(n_{\mathrm{cl}} \leq 100 \mathrm{~cm}^{-3}\right)$ the field energy can exceed the cloud kinetic energy and may "brake" the cloud's motion. A large molecular cloud may substantially deflect the jet motion, an event which might have occurred in the northern end of the jet (Plante et al. 1991).

Alignment of extragalactic jets with molecular gas is known for other sources and sometimes connected to star formation ("jet-induced star formation"). Examples are Centaurus A (e.g. Mould et al. 2000; Oosterloo \& Morganti 2005); Minkowski's object (e.g. Croft et al. 2006, and references therein), or highly redshifted quasars as BR 1202-0725 (Klamer et al. 2004). Recent observation of the latter example have shown that all the molecular gas in that source is concentrated in a compact nuclear course (Riechers et al. 2006). Jet-induced star formation 
is understood as mainly triggered by the gas shocked along the propagating jet (Oosterloo \& Morganti 2005).

So far, these models do not explain the over-abundance of molecular or atomic gas along the jets. Our model of jetambient medium interaction by ambipolar diffusion may explain the accumulation of molecular gas along the jets. In the case of NGC 4258 we observe for the first time the accumulation of CO gas along a jet in a nearby, spiral galaxy. Star formation, however, has not (yet) been triggered along these jets.

\section{Summary and conclusions}

Using the IRAM interferometer at Plateau de Bure, we mapped the ${ }^{12} \mathrm{CO}(1-0)$ emission of the central part of NGC 4258 along the nuclear jet direction in the inner $3 \mathrm{kpc}$. We further observed NGC 4258 in $\mathrm{H} \alpha$ at the Hoher List Observatory of the University of Bonn in order to ensure a proper relative positioning of the $\mathrm{H} \alpha$ and $\mathrm{CO}$ emission (Fig. 3). The observational results can be summarized as follows:

- We detected two parallel CO ridges along a PA of $-24^{\circ}$ with a total length of about $80^{\prime \prime}(2.8 \mathrm{kpc}$ ) (Fig. 2). The ridges are separated by a $\mathrm{CO}$ depleted funnel with a width of about $5^{\prime \prime}$ (175 pc).

- The $\mathrm{H} \alpha$ emission is more extended and broader than the $\mathrm{CO}$ emission with its maximum between the two $\mathrm{CO}$ ridges. Contrary to previous interpretations of Plante et al. (1991, 1994) we maintain that the CO does not outline "walls" around the $\mathrm{H} \alpha$ jets and hence does not confine it. It rather seems to be mixed in location and in velocity with the $\mathrm{H} \alpha$ gas.

- The CO iso-velocity map (Fig. 4) looks different from that of a "normal" spiral galaxy and indicates different velocity components which show up more clearly in the p-v diagrams along the galaxy's major axis (which is a good approximation for the orientation along the $\mathrm{CO}$ jet features) (Fig. 6) as parallel structures and in the pv-diagrams along the galaxy's minor axis (Fig. 7) as a blob structure. The main features in the p-v diagrams along the galaxy's major axis (Fig. 6) rise, however, linearly with distance from the nucleus with red shifted velocities in the northern half and blue shifted velocities in the southern half as expected for $\mathrm{CO}$ gas in galactic rotation.

- We further know from previous observations (Krause et al. 1990; Cox \& Downes 1996) that the molecular gas in NGC 4258 has only been detected in the center and along the $\mathrm{H} \alpha$ jets, at distances up to $2 \mathrm{kpc}$ (Krause et al. 1990). It has not been found in other parts of the (central) galactic disk.

We propose a model in which the northern jet points towards the observer (which also follows from the straightforward assumption that the jet is perpendicular to the nuclear accretion disk). In this case the CO emission is in normal but somewhat modified galactic rotation. The observed $\mathrm{H} \alpha$ may be former molecular gas which has been shock ionized during the partly violent interaction processes with the jets.

In our model the concentration of $\mathrm{CO}$ only along the ridges is due to an interaction of the rotating gas clouds with the jet's toroidal magnetic field (see Fig. 8) which increases the residence time of the molecular clouds near the jet when compared to other loci in the galactic disk. Both components, field and clouds, are coupled by ambipolar diffusion. We give some rough estimates of the kinematic time scales for the jet itself and the jet-cloud interaction on one hand and dynamical time scales of molecular clouds and the ambipolar diffusion on the other. The magnetic field strength used for the estimates are inferred from observations near the nucleus and further out up to kpc-scales together with theoretical jet models. We conclude that the proposed interaction is quite feasible from a theoretical point of view.

Concerning the observations, our model explains not only the concentration of $\mathrm{CO}$ along the jets and correspondingly the lack of $\mathrm{CO}$ elsewhere in the galactic disk. Furthermore it can explain the relative positions of the blobs within a single $\mathrm{p}-\mathrm{v}$ diagram perpendicular to the galaxy's major axis and between the various $\mathrm{p}-\mathrm{v}$ diagrams from north to south. The interaction of the molecular clouds with the jet's toroidal magnetic field (i.e. magnetic interaction) is comparable to the interaction of rotating gas with a spiral density wave (i.e. gravitational interaction) which is responsible for the appearance of the spiral arms in spiral galaxies.

In NGC 4258 we observed for the first time the accumulation of molecular gas along a jet in a nearby, spiral galaxy. A similar alignement of molecular gas along extragalactic jets is indicated in other more distant active galaxies and sometimes dicscussed in terms of "jet induced star formation". In NGC 4258, however, star formation has not (jet) been triggered along its jets.

Acknowledgements. It is a pleasure to thank Jörg Sanner and Tom Richtler from the Sternwarte of the University of Bonn for the $\mathrm{H} \alpha$ wide field images which were essential for the detailed comparison of $\mathrm{CO}$ and optical emission. We acknowledge discussions with Dennis Downes and Heino Falcke. We like to thank Phil Kronberg for fruitful discussions and helpful comments on the manuscript.

\section{References}

Adler, D. S.,\& Liszt, H. S. 1989, ApJ, 339, 836 van Albada, G. D. 1980, A\&A, 90, 123

van Albada, G. D., \& van der Hulst, J. M. 1982, A\&A, 115, 253

Blandford, R. D., Netzer, H., \& Woltjer, L. 1990, Active Galactic Nuclei (Heidelberg: Springer, 161)

Camenzind, M. 1990, Rev. Modern. Astron. 3, 234

Cecil, G., Wilson, A. S., \& Tully, R. B. 1992, ApJ, 390, 365

Cecil, G., Wilson, A. S., \& de Pree., C. 1995a, ApJ, 440,181

Cecil, G., Morse, J. A., \& Veilleux, S. 1995b, ApJ, 452, 613

Cecil G., Greenhill L. J., DePree, C. G. et al. 2000, ApJ 536, 675

Courtès, G., Cruvellier, P. 1961, C. R. Acad. Sci. Paris, 253, 218

Cox P., Downes D., 1996, ApJ, 473, 219

Croft, S., van Breugel, W., de Vries, W., et al. 2006, ApJ, 647, 1040

Daigle, A., \& Roy, J.-R. 2001, ApJ, 552, 144

Dettmar, R.-J., \& Koribalski, B. 1990, A\&A, 240, L15

Elmouttie, M., Krause, M., Haynes, R. F., \& Jones, K. L. 1998, MNRAS, 300, 1119

Falcke, H., \& Biermann, P. L. 1999, A\&A, 342, 49

Fendt, Ch., \& Cemeljic, M. 2002, A\&A, 395, 1045

Ferreira, J. 1997, A\&A, 319, 340

Gammie, C. F., Narayan, R., \& Blandford, R. D. 1999, ApJ, 516, 177

García-Burillo, S., Guélin, M., \& Cernicharo J. 1993, A\&A, 274, 148

García-Burillo, S., \& Guélin, M. 1995, A\&A, 299, 657

Greenhill, L. J., Jiang, D. R., Moran, J. M., et al. 1995, ApJ, 440, 619

Helfer T. T., Thornley M. D., Regan M. W., et al. 2003, ApJS, 145, 259

Herrnstein, J. R., Greenhill, L. J., \& Moran, J. M. 1996, ApJ, 468, L17

Herrnstein, J. R., Moran, J. M., Greenhill, L. J., et al. 1997, ApJ, 475, L17

Herrnstein, J. R., Greenhill, L. J., Moran, J. M., et al. 1998a, ApJ, 497, L69

Herrnstein, J. R., Moran, J. M., Greenhill, L. J., Blackman, E. G., \& Diamond, P. J. 1998b, ApJ, 508, 243

Herrnstein, J. R., Moran, J. M., Greenhill, L. J., et al. 1999, Nature, 400, 539

Herrnstein, J. R., Moran, J. M., Greenhill, L. J., \& Trotter, A. S. 2005, ApJ, 629, 719

Hyman, S. D., Calle, D., \& Weiler, K. W. 2001, ApJ, 551, 702

Hummel, E., Krause, M., \& Lesch, H. 1989, A\&A, 211, 266

Klamer I. J., Ekers R. D., Sadler E. M., \& Hunstead, R. W. 2004, ApJ, 612, L97 Krause, M., \& Löhr, A. 2004, A\&A, 420, 115

Krause, M., Beck, R., \& Klein, U. 1984, A\&A, 138, 385

Krause, M., Cox, P., García-Barreto, J. A., \& Downes, D. 1990, A\&A, 233, L1 
Krause, M., Löhr, A., Fendt, C., \& Neininger, N. 2005, in The Magnetized Plasma in Galaxy Evolution, ed. K. T. Chyzy, K. Otmianowska-Mazur, M. Soida, R.-J. Dettmar, Jagiellonian University, Kraköw, Poland van der Kruit, P. C., Oort, J. H., \& Mathewson, D. S. 1972, A\&A, 21, 169 Lada, C. J., \& Fich, M. 1996, ApJ, 459, 638

Martin, P., Roy, J-R., Noreau, L., \& LO, K. Y. 1989, A\&A, 345, 707

Miyoshi, M., Moran, J., Herrnstein, J., et al. 1995, Nature, 373, 127

Modjaz, M., Moran, J. M., Kondratko, P. T., \& Greenhill, L. J. 2005, ApJ, 626, 104

Mould, J. R., Ridgewell, A., Gallagher III, J. S., et al. 2000, ApJ, 536, 266

Neufeld, D. A., \& Maloney, P. R. 1995, ApJ, 447, L17

Oosterloo, T. A., \& Morganti, R. 2005, A\&A, 429, 469

Ouyed, R., \& Pudritz, R. E. 1997, ApJ, 482, 712

Parma, P., Fanti, C., Fanti, R., Morganti, R., \& de Ruiter, H. R. 1987, A\&A, 181, 244

Plante, R. L., Lo, K. Y., Roy, J-R., Martin, P., \& Noreau, L. 1991, ApJ, 381, 110

Plante, R. L., Handa T., \& Lo K. Y., 1994, in Astronomy with millimeter and submillineter wave interferometry, ed., Ishiguro M., Welch W. J. IAU Coll. 140, ASP Conf. Ser., 59, 359

Pringle, J. E., Antonucci, R. R. J., Clarke, C. J., et al. 1999, ApJ, 526, L9
Pudritz, R. E. 1990, ApJ, 350, 195

Regan, M. W., Thornley, M. D., Helfer, T. T., et al. 2001, ApJ, 561, 218

Riechers, D. A., Walter, F., Carilli, C. L., et al. 2006, ApJ, 650, 604

Sawada-Satoh, S., Ho, P. T. P., Muller, S., Matsushita, S., \& Lim, J. 2007, ApJ, 658,851

Shu, F. H. 1992, The physics of astrophysics, II. Gas dynamics (Sausalito: University Science Books)

Sofue, Y., Doi, M., Krause, M., Nakai, N., \& Handa, T. 1989, Publ. Astron. Soc. Japan, 41, 113

Sofue, Y. 1997, PASJ, 49, 17

Stahler, S. W. 1994, ApJ, 422, 616

Strong, A. W., Bloemen, J. B. G. M., Dame, T. M., et al. 1988, A\&A, 207, 1

de Vaucouleurs, G., de Vaucouleurs, A., \& Corwin, H. G. 1976, Second Reference Catalogue of Bright Galaxies (Austin: University of Texas Press)

Watson, W. D., \& Wallin, B. K. 1994, ApJ 432, L35

Weiß, A., Neininger, N., Hüttemeister, S., \& Klein, U. 2001, A\&A, 365, 571

Wilson, A. S., Yang, Y., \& Cecil, G. 2001, ApJ, 560, 689

Young, J. S., \& Scoville N. Z. 1991, ARA\&A, 29, 581

Yuan, F., Markoff, S., Falcke, H., \& Biermann, P. L. 2002, A\&A, 391, 139 